\title{
Berliner physiologische Gesellschaft
}

\section{Sitzung vom 24. Februar 1899}

$\mathrm{H}$ err 11 a m b u r ge r: W e it e re B e o b a c h t ungeii ü b e r d e n phy siologischen Pupillenabschluss.

Die neuen Experimente Hamburger's richten sicli gegen die ihni gemachten Einwände. Den Einwand, dass es unmöglicii sei be-stimint zu sagen, ob der Farbstoff in die hintere Kammer gebracht sei oder etwa versehentlicb in den Glaskörper, beseitigt er dadurch, dass er auf die gleiche Weise wie bei seinen Fluoresceïnversuchen Berliner Blau injicirt, das Ange sofort enucleirt und den Farbstoff nun mikro-skopiscb zwischen Iris und L-inse nachweist, also in der hinteren Kammer. Ferner liess II. die Spritze nac,li der Injection in situ stecken. sodass nicht nur jeder Flüssigkeitsabfluss unmöglich, sondern der Druck in der liintereu Kammer sogar offenbar stark vermehrt wurde, und obwohl das Auge hierbei luxirt gehalten werden muss - eine Stellung, welche nach H.'s früheren Versuchen zum Ueberlaufen der hinteren Kammer an sic·h schon Veranlassung gebeu kann, gelang es trotzdem aueh am nicht eserinisirten Thiere, den Farbstoff übertvitt 5 Minuten lang zu verzögern. Audi das physiologiscbe Pupillenspiel braucht keine Communication zu bedingen; 5 Minuten langes abwecbselndes Beleuchten und Beschatten des Auges lookte den Farbstoff nicht her-bei, obwohl die Pupille hier von 8 auf 3 mm. zusammenschnurrte. Seine Versuche an Albinos halt II. í'iir besonders werthvoll, weil die pigmentfreie Iris deutlich erkennen lässt, dass der Farbstoff sicli eher ringförmig in der hinteren Kammer ausbreitet, als dass er den kurzen Weg ins Pupillargebiet findet: dieser Weg ist ja eben durch den Sphincter iridis versperrt, der als Scbliessmuskel einen Tonus ausübt und für seinen Druck an der frei vorragenden Linsenconvexität gleich-sam ein Widerlager findet. so dass Iris und Linse wasserdiclit von einander adaptirt bleiben. Ein Experimen†um crucis bilden 6 Versuche an iridectomirten Thieren, bei denen sofort der Farbstoffüber-tritt, an einem Colobomschenkel erfolgt. Den physiologischen Pupillen-abschlvss will aber II. nicht für einen hermetischen, absolut eonstanten aufgeíasst wissen: denn er kann durchbrochen werden bei maximaler Mydiasis, Entzüudungen der Iris und des Corpus ciliare, beim Menschen vielleicht auch bei jeder stärkeren Hyperämie des Auges.

Herr Turk: Untersuchunge u über die Entstehung des phy siologischen Netzhautvenenpulses. T. beobachtete bei einer Anzahl Personen mit unregelmässiger Herzaction dass beim Ausbleiben einer arteriellen Pulswelle die pul-sirenden papillaren Xetzhautvenen-Enden nach ihrer dem letzten regel-mässigen Radialj > ulse folgenden Erweiterung sicli wie sonst, nur meist etwas starker, verengten und $\mathrm{w}^{3} / 4 \mathrm{hrend}$ der ganzen Zeit der ausfalleuden arteriellen Pulswelle verengt blieben; die Erweiterung folgte erst wieder dem nächsten Radialpulse. Die Herzsystole bewirke also bei der Pulsation der Venen-Enden die Erweiterung, nicht die Verengerung. Diese Erweiterung konne nicht durch rhythmische Erschwerung des Blutabflusses bedingt sein, da sicli experimentell nachweisen lässt, dass

408

Sitzungsberichte. 
eine auf letztere Art entstehende Erweiterung in einem elastischen Schlauche sich iramer entgegen der Stromrichtung ausbreite. Die pulsatorisclie Erweiterung der Netzhautvenen-Enden erfolge aber in der Stromrichtung: danacli bleibe nur anzunehmen, das.s diese Erweiterung durch gesteigerten peripheren Blutzufluss zu den Venen entstehe, d. h. dass die arterielle Pulswelle sich durch die Capillaren ins in die Venen der Retina verbreite. Die zwischen den pulsatorischen Erweiterungen bestehende Verengerung der Venen-Enden sei eine Erscheinung, die, wie aus den Versuchen von Koerner und Klernensiewicz zu schliessen, in jedem dünnwandigen nachgiebigen Strömungsrohre am Ende eines unter äusseren Druck stehenden Bezirkes auftreten und vnter gewissen Bedingungen als dauernder Gleichgewichtszustand be-stehen kann.

Den Grund fur die besonders weite Ausbreitung der Pulswellen in den Netzhautgefässen sieht Turk in den relativ hohen extravascu-lären (intraocularen) Drucke, der auf diesen Gefässen lastet, eine Auf-fassung, welche gestützt wird durch Beobachtnngen von Roy und Brown an der Froschschwimrnhaut, welche Turk nur bestätígen kaon. Als Grund dafür, dass die Pulswelle bei ihrem Gauge durch die Netz-hautgefässe erst in den Enden der Venen in deut Пchere Erscheinung trete, sei die zwischen den Pulsen bestehende starke Verengerung dieser Enden anzusehen, da an verengten Stellen einer Strombahn ein pulsatorischer Flüssigkeitszuwachs eine relativ stärkere Erweiterung ver-ursachen müsse. T. halt fiir möglich, dass auch in anderen Organen mit hohem Ge $\Lambda$ vebsdruck ein ,, progressive!-" Venenpuls physiologischer Weise bestehe. Vielleicht sei auch der Hirnvenenpuls als solcher auf-zufassen. -

Berlinep Gesellschaft füp Psyehiatpie und Nervenkpankheiten.

Sitzung yo $\pi$ i 13. März 1899.

Herr $\Lambda$ V. König: Ueber ,springende Pupillen” in einem Falle von cerebraler Kinderlähmung nebst einig'en Be-merkungen über die prognostische Bedeutung der ,sprin-genden Pupillen” bei normaler L· ichtreaction.

Der Vortragende fasst seine Ausführungen folgendermassen z $\alpha$-sarnmen:

1. Die ,springende Mydriasis” ist eine seltene Erscheinung. Siekommt bei normaler wie pathologischer Pupillenreaction vor. Beob-achtet wnrde sie bisher hauptsächlich bei organischen Erkrankungendes Central-Nervensystems, selten bei soldier functioneller Natur, undnur vereinzelt bei Nervenleidenden resp. sonst Gesunden.

Die prognostische Bedeutung der ,springenden” Mydriasis kommt nur bei normaler Pupillenreaction in Betracht, da bei jiatho-logischer Pupillenreaction dies*1 Thatsache allein die nöthige Directive geben wird. 